\title{
A prediction model for placenta accreta spectrum: A multicentre external validation study.
}

\author{
Shubhangi Singh ${ }^{1}$, Daniela Carusi ${ }^{2}$, Penny Wang ${ }^{2}$, Elena Reitman-Ivashkov ${ }^{3}$, Ruth \\ Landau $^{3}$, Kara Fields ${ }^{2}$, Carolyn Weiniger ${ }^{4}$, and Michaela Farber ${ }^{2}$ \\ ${ }^{1}$ University of Michigan \\ ${ }^{2}$ Brigham and Women's Hospital \\ ${ }^{3}$ Columbia University \\ ${ }^{4}$ Tel Aviv Sourasky Medical Center
}

April 7, 2021

\begin{abstract}
Objective: To validate the Weiniger model, a multivariable prediction model for placenta accreta spectrum (PAS). Design: Multicentre external validation study. Setting: Two tertiary care hospitals in the United States. Population: Cohort A included patients with risk factors (prior caesarean delivery, placenta praevia) and/or ultrasound features of PAS (variable risk) presenting to a tertiary care hospital. Cohort B patients were referred to a tertiary care hospital specifically for ultrasound features of PAS (higher risk). Methods: Weiniger model variables (prior caesarean deliveries, placenta praevia and ultrasound features of PAS) were retrospectively collected from both cohorts and predictive performance of the model was evaluated. Main Outcome Measures: Surgical and/or pathological diagnosis of PAS. Results: The model c-statistic in cohorts A and B was 0.728 (95\% CI: $0.662,0.794)$ and 0.866 (95\% CI: 0.754, 0.977) signifying acceptable and excellent discrimination, respectively. Based on calibration curves, the model underestimated average PAS risk in both cohorts. In both cohorts, high risk was overestimated and low risk underestimated. Use of this model compared to a "treat all" strategy had greater net benefit at a threshold probability of $>0.25$ in cohort A, but no net benefit in cohort B. Conclusions: This study provides multicentre external validation of the Weiniger model for PAS prediction, making it a useful triaging tool for management of this high-risk obstetric condition. Clinical usefulness of this model is influenced by the incidence of risk factors and PAS ultrasound features, with better performance in a variable-risk population at threshold probability $>25 \%$.
\end{abstract}

Title: A prediction model for placenta accreta spectrum: A multicentre external validation study.

Author Information: Shubhangi Singh, $\mathrm{MD}^{1,2}$; Daniela A. Carusi, $\mathrm{MD}^{3}$; Penny Wang, $\mathrm{BA}^{3}$; Elena ReitmanIvashkov, MD ${ }^{4}$; Ruth Landau, MD ${ }^{4}$; Kara G. Fields, $\mathrm{MS}^{1}$; Carolyn F. Weiniger, MB $\mathrm{ChB}^{5}$; Michaela K. Farber, $\mathrm{MD}^{1}$.

${ }^{1}$ Department of Anesthesiology, Perioperative and Pain Medicine, Brigham and Women's Hospital, Harvard Medical School, Boston, MA, USA

USA

${ }^{2}$ Present address: Department of Anesthesiology, University of Michigan Medical School, Ann Arbor, MI,

${ }^{3}$ Department of Obstetrics and Gynecology, Brigham and Women's Hospital, Harvard Medical School, Boston, MA, USA.

${ }^{4}$ Department of Anesthesiology, Columbia University College of Physicians and Surgeons, New York, NY, USA. 
${ }^{5}$ Division of Anaesthesia, Critical Care and Pain, Tel Aviv Sourasky Medical Center, Tel Aviv, Israel

Corresponding Author:

Shubhangi Singh, MD

Department of Anesthesiology, University of Michigan Medical School

1500 E Medical Center Drive, Ann Arbor, MI-48109, USA

Phone number: (734) 763-2436

Fax number: (734) 763-3354

Email: shsi@med.umich.edu

Shortened running Title: External Validation of a PAS Prediction Model.

\section{ABSTRACT}

Objective: To validate the Weiniger model, a multivariable prediction model for placenta accreta spectrum (PAS).

Design: Multicentre external validation study.

Setting: Two tertiary care hospitals in the United States.

Population: Cohort A included patients with risk factors (prior caesarean delivery, placenta praevia) and/or ultrasound features of PAS (variable risk) presenting to a tertiary care hospital. Cohort B patients were referred to a tertiary care hospital specifically for ultrasound features of PAS (higher risk).

Methods: Weiniger model variables (prior caesarean deliveries, placenta praevia and ultrasound features of PAS) were retrospectively collected from both cohorts and predictive performance of the model was evaluated.

Main Outcome Measures: Surgical and/or pathological diagnosis of PAS.

Results: The model c-statistic in cohorts A and B was 0.728 (95\% CI: 0.662, 0.794) and 0.866 (95\% CI: $0.754,0.977)$ signifying acceptable and excellent discrimination, respectively. Based on calibration curves, the model underestimated average PAS risk in both cohorts. In both cohorts, high risk was overestimated and low risk underestimated. Use of this model compared to a "treat all" strategy had greater net benefit at a threshold probability of $>0.25$ in cohort A, but no net benefit in cohort B.

Conclusions: This study provides multicentre external validation of the Weiniger model for PAS prediction, making it a useful triaging tool for management of this high-risk obstetric condition. Clinical usefulness of this model is influenced by the incidence of risk factors and PAS ultrasound features, with better performance in a variable-risk population at threshold probability $>25 \%$.

Tweetable abstract: This multi-centre study externally validates a model for prediction of placenta accreta among at-risk women.

Keywords: abnormal placentation, placenta increta, placenta percreta, Weiniger model, ultrasound, caesarean delivery, placenta praevia, placental implantation, invasive placenta, infiltrative placenta.

\section{INTRODUCTION}

Placenta accreta spectrum (PAS) describes a range of pathologic placental attachment to and invasion of the uterine myometrium and includes placenta accreta, increta, and percreta. ${ }^{1} \mathrm{PAS}$ is a leading cause of severe postpartum haemorrhage (PPH) and emergent hysterectomy. ${ }^{2}$ Management of PAS and associated maternal morbidity, preterm birth and maternal and neonatal intensive care unit (ICU) admission can be optimised at tertiary level hospitals with specialised multidisciplinary care. ${ }^{3}$ Anticipatory planning with recognition of 
risk factors for PAS is critical to allow transfer to an appropriate delivery centre, allocate necessary resources and minimise life-threatening PPH and associated complications. ${ }^{4-6}$

Early recognition of suspected PAS is facilitated by identification of risk factors including placenta praevia (praevia), prior caesarean delivery (CD), multiparity, prior uterine surgeries, uterine anomalies, in vitro fertilisation and advanced maternal age..$^{7-11}$ Diagnostic confirmation of PAS can only happen at delivery, as radiographic evaluation by ultrasound (US) or magnetic resonance imaging (MRI) alone does not reliably predict or refute the presence of PAS. ${ }^{6,12-14}$

A PAS prediction model by Weiniger et al. was derived from analysis of 92 deliveries based on number of prior CDs, presence of praevia, and US features of PAS. ${ }^{9}$ The model had good discrimination by area under the curve (AUC) and may be more generalisable, comprehensive, and easier to use than alternative PAS models. ${ }^{15-23}$ We aimed to validate this model in two cohorts derived from two tertiary-care hospitals: a variable-risk cohort that included patients with risk factors (prior CD and praevia) and/or US features of PAS (Cohort A) and a higher-risk referral population all of who had US features of PAS and were referred to the PAS service of a tertiary care hospital. Our hypothesis was that this model would demonstrate external validity among the obstetric patients in both tertiary care settings.

\section{MATERIALS AND METHODS}

This manuscript follows the Transparent Reporting of a multivariable prediction model for Individual Prognosis or Diagnosis (TRIPOD) guidelines. ${ }^{24}$ The model was previously developed from a prospective cohort (henceforth referred to as the Weiniger development cohort) at the Hadassah Hebrew University Medical Center, a tertiary-care hospital in Israel..$^{9}$ Briefly, all women with praevia and/or prior history of CD were screened for US features of PAS (Table S1). Women with both high- and low-risk features were included. Women who had no US features of PAS, but had both praevia and prior CD were also included (Figure 1A). ${ }^{9}$ Using the same inclusion criteria, validation cohort A (cohort A) was retrospectively constituted from a database of all deliveries from 2007-2017 at Brigham and Women's Hospital, a tertiary hospital in Boston, MA, United States (Figure 1B). Validation cohort B (cohort B) was retrospectively constituted from a database of patients referred, based on any US features of PAS, to Columbia University Irving Medical Center, a tertiary hospital in New York, NY, United States (Figure 1C).

\section{Data collection}

Maternal age, gravidity, parity, number of prior CDs, current praevia, US features of PAS, confirmed PAS at delivery, hysterectomy, and placental pathology reports were retrospectively collected by chart review in both cohorts.

\section{Estimation of risk}

PAS risk was estimated from number of prior CDs $(N)$, presence of praevia $(R ; 1=$ yes, $0=$ no) and US features of PAS ( $H ; 1=$ high-risk US features, $0=$ low-risk US features). The risk score (probability index, $P$ ) was calculated for each woman using the following equation as derived in the model development paper: ${ }^{9}$

$P=\mathrm{e}^{Y} /\left(1+\mathrm{e}^{Y}\right)$

Where $\mathrm{Y}$ is $\log$ odds of having PAS and is the linear function of the independent risk factors, calculated using the equation:

$Y=-8.2862+(6.5184 \mathrm{x} R)+(2.3313 \mathrm{x} N)+(2.7272 \mathrm{x} H)-(2.1151 \times \mathrm{x} N R)$.

\section{US features of PAS $(H)$}

US features of PAS were classified as high- or low-risk as defined by Weiniger et al. (Table S1). ${ }^{9}$ If PAS could not be ruled out per sonographic report, the patient was classified as "low-risk." Women with no US features but with history of prior CD and current praevia were also classified as low-risk for model input per Weiniger development cohort criteria. 


\section{Outcome}

The outcome variable was clinical or pathological confirmation of PAS at delivery. Clinical confirmation of PAS was made by the surgeon's report of visible PAS or focal accreta, difficulty creating a plane between the placenta and endometrial wall, or adherence or excessive bleeding with attempted placental removal. If clinical and pathological confirmation of PAS were inconsistent with each other, the diagnosis of PAS was maintained.

\section{Statistical analysis}

Magnitude and direction of differences in patient characteristics and outcomes between each validation cohort and the Weiniger development cohort were quantified as standardized differences. For continuous, ordinal categorical, and nominal categorical variables, standardized differences were calculated as the difference in means, ranks, and proportions between groups, respectively, divided by the pooled within-group standard deviation (SD). ${ }^{25}$ A standardized difference $>0.1$ was used as the threshold for notable difference between groups. ${ }^{26}$ We implemented the framework of Debray et al. ${ }^{27}$ to determine whether the overall case-mix differences between the Weiniger development and each validation cohort were consistent with reproducibility (model performance in the same target population) or transportability (model performance in different but related populations). C-statistic of membership models, ratio of SD of linear predictor (LP) and estimated difference in mean LP between the Weiniger development and validation cohorts were calculated for this purpose (Appendix S1).

Model performance in each validation sample was evaluated with respect to discrimination, ${ }^{28,29}$ calibration, ${ }^{30}$ and clinical usefulness ${ }^{31}$ which are described in detail in appendix S2. Discrimination was evaluated in each validation cohort by plotting receiver operating characteristic (ROC) curves, estimating the area under ROC curve via c-statistic with $95 \%$ Wald confidence intervals (CI) ${ }^{28}$ and estimating sensitivity, specificity, positive predictive value (PPV), and negative predictive value (NPV) at two thresholds presented by Weiniger. ${ }^{9}$ Calibration was evaluated by plots of observed versus predicted probability of PAS in each population and the corresponding regression intercepts and slopes $^{30}$. $95 \%$ CI for calibration curve intercepts and slopes were estimated using the bias-corrected and accelerated bootstrap method with 1000 bootstraps. Clinical usefulness $^{31}$ of the model was assessed via decision curve analysis with plots of net benefit (i.e., net truepositive classification rate) of (1) assuming all patients are low-risk (treat-none) (2) assuming all patients are high-risk (treat-all), and (3) employing the model ${ }^{32}$ over a range of threshold probabilities (i.e. the probability of having PAS at which the clinicians would change their management strategy). Visual comparison of these curves indicated the threshold probability at which risk prediction with the model had a greater net benefit than assuming all patients are high risk. Net benefit was also compared between the prediction model and treat-all strategies specifically at 3 predetermined thresholds: 10\%, 30\%, and $50 \%$ predicted risk. $95 \%$ CI at thresholds were estimated using the bias-corrected and accelerated bootstrap percentile method with 5000 bootstraps. Statistical analyses were performed using SAS software version 9.4 (SAS Institute, Cary, NC) and R software version 3.6.1 (R Foundation for Statistical Computing, Vienna, Austria.

\section{RESULTS}

\section{Baseline characteristics and outcomes:}

The Weiniger development cohort, cohort A, and cohort B included 92, 253, and 99 patients respectively (Figure 1). No data were missing for model outcomes or predictors.

In Weiniger development cohort, $56.5 \%(\mathrm{n}=52$ of 92$)$ of suspected cases were confirmed to have PAS at delivery. Similarly, $51.8 \%$ of suspected cases $(\mathrm{n}=131$ of 253$)$ in cohort A were confirmed to have PAS. Of the 131 patients with confirmed PAS in cohort A, 126 (96.2\%) were diagnosed by clinical criteria of which 98 $(77.7 \%)$ were confirmed by pathology. Five cases without a clinical diagnosis in cohort A were confirmed by histopathology to have PAS. In contrast, $93.9 \%$ of suspected cases $(n=93$ of 99$)$ in cohort B were confirmed to have PAS at delivery (Table 1). Eighty-two of these cases $(88.2 \%)$ were confirmed pathologically. None of the cases in cohort B had positive pathology in absence of PAS by clinical diagnosis. 
Compared to the Weiniger development cohort, median gravidity and parity were lower in both validation cohorts, mean age was higher, and hysterectomy with confirmed PAS was higher in cohort B and lower in cohort A (Table 1). The distribution of individual model predictors in cohort A was similar to the Weiniger development cohort except for median number of prior CDs, which was lower in cohort A. Cohort B had higher prevalence of high-risk US features compared to the Weiniger development cohort but had similar distribution of other model predictors (Table 1).

\section{Relatedness of the development and validation cohorts:}

Membership model c-statistics for cohorts A and B vs. the Weiniger development cohort were 0.845 (95\% CI: $0.793,0.897)$ and 0.883 (95\% CI: 0.835, 0.931), respectively (Table 2). This indicated that patients in each validation cohort can be strongly discriminated from the Weiniger development cohort based on patient characteristics and observed PAS outcome. The mean \pm SD LP was $0.23 \pm 1.96$ in Weiniger development cohort, $-0.60 \pm 2.09$ in cohort $\mathrm{A}$, and $0.80 \pm 1.37$ in cohort B. The ratio $(95 \% \mathrm{CI})$ of the LP SD between cohort A and Weiniger development cohort was 1.07 (0.99, 1.47), indicating a similar degree of case-mix heterogeneity. In contrast, the ratio of the LP SD between cohort B and Weiniger development cohort indicated less case-mix heterogeneity in cohort B vs. Weiniger development cohort (ratio [95\% CI]: 0.71 $[0.36,0.78])$. The difference $(95 \%$ CI) in LP mean between cohort A and Weiniger development cohort was $-0.83(-1.32,-0.34)$, indicating a lower average predicted risk of PAS in cohort A. In contrast, the difference in LP mean between cohort B and Weiniger development cohort indicated a higher average predicted risk in cohort B (difference in means [95\% CI]: $0.57[0.09,1.06]$ ). These overall differences in the patient case mix between the two validation cohorts and Weiniger development cohort indicated that validation of the model in these external samples is assessing transportability.

\section{Model performance}

\section{Discrimination}

ROC curves for the Weiniger development and both validation cohorts are shown in Figure 2. A model c-statistic of 0.846 (95\% CI $0.758,0.933)$ was observed in the Weiniger development dataset, corresponding with excellent discrimination. A c-statistic of 0.728 (95\% CI: $0.662,0.794)$ was observed when the model was applied to cohort A, corresponding with acceptable discrimination. A c-statistic of 0.866 (95\% CI: 0.754 , 0.977) was observed in cohort B, corresponding with excellent discrimination (Table 3).

\section{Calibration}

Plots of predicted versus observed probability of PAS for all cohorts are shown in Figure 3. Table 3 presents intercepts and slopes for these calibration curves. Model calibration was optimal in the development sample, as demonstrated by an intercept of 0 (95\% CI: $-0.589,0.646)$ and a slope of 1.000 (95\% CI: 0.629, 1.428). In contrast, the intercepts for cohort A $(0.266 ; 95 \%$ CI: 0.004, 0.506) and B $(2.636 ; 95 \%$ CI: 1.733, 3.741), both $>0$, indicated that risks were underestimated on average in these external samples as described in appendix S2. The slopes for cohort A $(0.342 ; 95 \%$ CI: $0.170,0.532)$ and B $(0.604 ; 95 \%$ CI: $-0.166,1.221)$, both $<1$ indicated overfitting of the model for both validation cohorts, which translates to overestimation of high risk and underestimation of low risk (Appendix S2).

\section{Clinical usefulness}

Figure 4 shows plots of net benefit at various threshold probabilities for the strategies of (1) assuming all patients are low-risk (treat-none), (2) assuming all patients are high-risk (treat-all), and (3) using the model. In the Weiniger development cohort, use of the model corresponded with net benefit equal to or greater than the treat-all strategy across all threshold probabilities (Figure 4A). In cohort A, using the model corresponded with higher net benefit than the treat-all strategy starting at a threshold probability of 0.25 (Figure 4B). In cohort B, the treat-all strategy had a greater net benefit than using the model across all threshold probabilities (Figure 4C; Table 4).

\section{DISCUSSION:}




\section{Main findings:}

This study provides external validation of the Weiniger model for PAS prediction in two distinct patient populations. The Weiniger model performed well in both cohorts A and B with acceptable and excellent discrimination, respectively, suggesting reliability to identify women with PAS in both populations, transportability and generalizability, and applicability to populations that meet criteria for PAS suspicion but are otherwise different from the development cohort.

The clinical usefulness of the model to identify women with PAS is dependent on the risk of the population, with more utility in a variable-risk, heterogenous population of patients with prior CD, current praevia, and/or presence of US features of PAS (Cohort A, Figure 4B) compared to a higher-risk, homogenous population in which all patients had US features of PAS (Cohort B, Figure 4C). Model performance was enhanced when at-risk patients without US findings were included, compared to only including women with US risk factors for PAS. Since there were differences between cohorts A and B in the proportion of women with confirmed PAS ( $52 \%$ vs $94 \%$ ), baseline characteristics, distribution of model predictors (Table 1) and membership model performance (Table 2), a combined analysis was not undertaken.

In cohort $\mathrm{A}$, the net benefit depended on the probability threshold of the clinician for determining delivery timing, location and multidisciplinary planning. The model became more useful than a treat-all strategy above a probability threshold of $25 \%$; if a likelihood of having PAS $>1$ in every 4 patients would prompt the clinician to change their management, prediction of PAS using the model was more beneficial. However, if the threshold for a particular decision or intervention was $<10 \%$ (for example, the threshold to refer a patient with suspected PAS to a high-resource setting), it was more reasonable to treat every patient with the model risk factors as if they had PAS.

\section{Strengths and limitations:}

There are some advantages of the Weiniger model when compared to other models (Table S2). The Weiniger model utilizes US as opposed to MRI. ${ }^{19-21}$ US is more readily available, and the superiority of MRI over US for diagnosis of PAS has not been established. ${ }^{33-36}$ Risk of PAS increases with each CD, with a 10-fold increase of concurrent PAS with 5 vs. 2 prior CDs. ${ }^{37}$ The Weiniger model accounts for this by integrating the total number of prior CDs as a predictor, rather than having a cut off (e.g. [?]2 or 3) for scoring CDs. ${ }^{15-18,21}$ The Weiniger model was developed using a comparatively lower-risk population compared to several other models (Table S2) ${ }_{15,17-21,23}$ which may make it more generalizable, as confirmed by the current study. Of note, a model developed and internally validated by Yang et $\mathrm{al}^{22}$ in a large cohort of over 8000 patients had high discrimination of 0.93 and was based solely on clinical characteristics (prior CD, vaginal bleeding, medications during pregnancy, praevia). Although the Yang model may be useful in community settings, it does not include US features of PAS which can be reliably assessed in high-resource settings.

Another strength of this study is that it is a large multi-centre analysis to externally validate a PAS prediction model in two cohorts that were dissimilar to the Weiniger development cohort and to each other. The results suggest good external validity. The study not only evaluated the calibration, discrimination, sensitivity and specificity, but also the clinical usefulness of the model in the form of a decision curve analysis. We further derived the net benefit at different probability thresholds to establish a user-friendly tool for practical management and decision-making based on available resources. We specifically demonstrated that the model is most useful above a threshold probability of $25 \%$ in a variable-risk population.

This study is not without limitations. Neither the Weiniger development nor validation cohorts included women who had PAS in the absence of risk factors which may constitute $>50 \%$ of PAS in the community, so use of the model should not preclude readiness for unanticipated PAS cases. ${ }^{38}$ Second, the validation cohorts spanned a significant duration (11 and 7 years), during which diagnostic and management practices may have changed significantly. Another limitation is the retrospective nature of the validation cohorts. Given that PAS is a rare condition, prospective enrolments of large cohorts would be difficult and timeconsuming. However, reliable retrospective data extraction was possible because the features evaluated for cohort inclusion are routinely documented in the medical record. Datasets with a minimum of 100 events 
and 100 non-events are recommended for external validation of prediction models developed using logistic regression. ${ }^{39}$ Given the rarity of PAS, despite the long duration of data collection, this recommendation was met in cohort A but not in cohort B. Therefore, model performance estimate precision in cohort B may be suboptimal. In our model, US features were scored in a binary fashion. This approach may have lowered the precision of the model for determining the risk of specific features compared to some other models available in the literature. ${ }^{15-18,23}$

\section{Interpretation:}

Use of this model for decision-making depends on the ability to escalate resources in response to PAS at the time of CD. In high-resource settings, using the model may allow deployment of treatment modalities at different probability thresholds. For example, while the threshold for alerting interventional radiology (IR) may be low (e.g., 30\%), it may be reasonable to have a higher treatment threshold, e.g., 50\%, for having IR personnel in the operating room. In contrast, low-resource hospitals may need to use a lower threshold $(<25 \%)$ for referral to a specialist service or transfer to higher level of maternal care and may benefit by treating all patients with inclusion criteria as high-risk. Similarly, there may be different treatment thresholds for different anaesthetic modalities and types of vascular access (arterial, central and occlusive balloon catheter access).

\section{Conclusions:}

This validation study demonstrates that the Weiniger model for PAS prediction successfully identified patients with PAS in two distinct patient populations in the United States. In low resource settings and in referral populations with a high prevalence of PAS, it is likely be beneficial to treat every at-risk patient as having PAS. However, at high resource centres managing general obstetric populations, this model may be helpful to triage patients with variable risk of PAS. Use of this model may refine communication and planning for patients with suspected PAS. Of note, the NPV of this model was 0.722 in cohort A at the optimal cut off. This suggests that $27.8 \%$ of the women predicted to nothave PAS by the model would actually have PAS, highlighting the importance of having emergency protocols in place. Centres must also recognize the inherent possibility of unanticipated PAS among women with no risk factors and have systems for managing such emergencies.

Although this model can predict the presence of PAS, it cannot predict the degree of invasion or severity of haemorrhage, nor the surgical complexity of an individual case. Thus, research toward models that predict clinically relevant measures including blood loss, ICU requirement, and maternal morbidity are warranted to further refine surgical and anaesthetic planning for patients at risk for PAS is warranted.

Acknowledgments : We thank and acknowledge Lisa Deutsch PhD, BioStats, Modiin, Israel for her assistance with the statistical analysis for one of the posters presented at the $51^{\text {st }}$ Annual meeting of the Society for Obstetric Anesthesia and Perinatology held from May $1^{\text {st }}-5^{\text {th }} 2019$ in Phoenix, Arizona.

Disclosure of Interests: The authors report no conflict of interests.

\section{Authors Contributions:}

SS: Study design, Data collection at Brigham and Women's Hospital, analysis planning, manuscript preparation.

DC: Data collection at Brigham and Women's Hospital and manuscript preparation.

PW: Data collection at Brigham and Women's Hospital

ERI: Study design and data collection at Columbia University Irving Medical Center.

RL: Study idea and design, data collection at Columbia University Irving Medical Center, manuscript preparation.

KF: Data analysis 
CW: Study idea, manuscript preparation

MF: Study idea, design, manuscript preparation.

Details of Ethics Approval: The study was approved by the institutional review board (IRB) of both Brigham and Women's Hospital (IRB number: 2018P003126 ; date of approval: December $24^{\text {th }}, 2018$ ) and Columbia University Irving Medical Center (IRB number: AAAD3960; date of approval: July 15 $5^{\text {th }}$, 2008 and last renewed on April 30 $30^{\text {th }}, 2019$ ) with waivers of informed consent.

Funding: Support was provided solely from institutional and/or departmental sources.

\section{REFERENCES:}

1. Abuhamad A. Morbidly adherent placenta. Semin Perinatol. 2013 Oct;37(5):359-64.

2. Committee on Practice Bulletins-Obstetrics. Practice Bulletin No. 183: Postpartum Hemorrhage. Obstet Gynecol. 2017;130(4):e168-86.

3. Farquhar CM, Li Z, Lensen S, McLintock C, Pollock W, Peek MJ, et al. Incidence, risk factors and perinatal outcomes for placenta accreta in Australia and New Zealand: a case-control study. BMJ Open. 2017 Oct 5;7(10):e017713.

4. Silver RM, Fox KA, Barton JR, Abuhamad AZ, Simhan H, Huls CK, et al. Center of excellence for placenta accreta. Am J Obstet Gynecol. 2015 May;212(5):561-8.

5. Jauniaux E, Bhide A, Kennedy A, Woodward P, Hubinont C, Collins S. FIGO consensus guidelines on placenta accreta spectrum disorders: Prenatal diagnosis and screening,. International Journal of Gynecology \& Obstetrics. 2018;140(3):274-80.

6. Society of Gynecologic Oncology, American College of Obstetricians and Gynecologists and the Society for Maternal-Fetal Medicine, Cahill AG, Beigi R, Heine RP, Silver RM, et al. Placenta Accreta Spectrum. Am J Obstet Gynecol. 2018;219(6):B2-16.

7. Beuker JM, Erwich JJHM, Khong TY. Is endomyometrial injury during termination of pregnancy or curettage following miscarriage the precursor to placenta accreta? J Clin Pathol. 2005 Mar;58(3):273-5.

8. Fitzpatrick KE, Sellers S, Spark P, Kurinczuk JJ, Brocklehurst P, Knight M. Incidence and Risk Factors for Placenta Accreta/Increta/Percreta in the UK: A National Case-Control Study. PLoS One [Internet]. 2012 Dec 27 [cited 2020 Mar 7];7(12). Available from: https://www.ncbi.nlm.nih.gov/pmc/articles/PMC3531337/

9. Weiniger CF, Einav S, Deutsch L, Ginosar Y, Ezra Y, Eid L. Outcomes of prospectively-collected consecutive cases of antenatal-suspected placenta accreta. Int J Obstet Anesth. 2013 Nov;22(4):273-9.

10. Kyozuka H, Yamaguchi A, Suzuki D, Fujimori K, Hosoya M, Yasumura S, et al. Risk factors for placenta accreta spectrum: findings from the Japan environment and Children's study. BMC Pregnancy Childbirth [Internet]. 2019 Nov 27 [cited 2020 Mar 7];19. Available from: https://www.ncbi.nlm.nih.gov/pmc/articles/PMC6882023/

11. De Mucio B, Serruya S, Aleman A, Castellano G, Sosa CG. A systematic review and meta-analysis of cesarean delivery and other uterine surgery as risk factors for placenta accreta. Int J Gynaecol Obstet. 2019 Dec;147(3):281-91.

12. D'Antonio F, Iacovella C, Bhide A. Prenatal identification of invasive placentation using ultrasound: systematic review and meta-analysis. Ultrasound Obstet Gynecol. 2013 Nov;42(5):509-17.

13. Bhide A, Sebire N, Abuhamad A, Acharya G, Silver R. Morbidly adherent placenta: the need for standardization. Ultrasound Obstet Gynecol. 2017;49(5):559-63.

14. Satija B, Kumar S, Wadhwa L, Gupta T, Kohli S, Chandoke R, et al. Utility of ultrasound and magnetic resonance imaging in prenatal diagnosis of placenta accreta: A prospective study. Indian J Radiol Imaging. 
2015 Dec;25(4):464-70.

15. Rac MWF, Dashe JS, Wells CE, Moschos E, McIntire DD, Twickler DM. Ultrasound predictors of placental invasion: the Placenta Accreta Index. Am J Obstet Gynecol. 2015 Mar;212(3):343.e1-7.

16. Tovbin J, Melcer Y, Shor S, Pekar-Zlotin M, Mendlovic S, Svirsky R, et al. Prediction of morbidly adherent placenta using a scoring system. Ultrasound Obstet Gynecol. 2016 Oct;48(4):504-10.

17. Marsoosi V, Ghotbizadeh F, Hashemi N, Molaei B. Development of a scoring system for prediction of placenta accreta and determine the accuracy of its results. J Matern Fetal Neonatal Med. 2018 Nov 4;1-7.

18. Luo L, Sun Q, Ying D, Wu X, Yan P, Yang Y, et al. Scoring system for the prediction of the severity of placenta accrete spectrum in women with placenta previa: a prospective observational study. Arch Gynecol Obstet. 2019 Sep;300(3):783-91.

19. Sun H, Qu H, Chen L, Wang W, Liao Y, Zou L, et al. Identification of suspicious invasive placentation based on clinical MRI data using textural features and automated machine learning. Eur Radiol. 2019 Nov;29(11):6152-62.

20. Delli Pizzi A, Tavoletta A, Narciso R, Mastrodicasa D, Trebeschi S, Celentano C, et al. Prenatal planning of placenta previa: diagnostic accuracy of a novel MRI-based prediction model for placenta accreta spectrum (PAS) and clinical outcome. Abdom Radiol (NY). 2019 May;44(5):1873-82.

21. Chu C, Zhao S, Ding M, Liu M, Zhang Y, Bao L, et al. Combining Clinical Characteristics and Specific Magnetic Resonance Imaging Features to Predict Placenta Accreta. J Comput Assist Tomogr. 2019 Oct;43(5):775-9.

22. Yang T, Li N, Qiao C, Liu C. Development of a Novel Nomogram for Predicting Placenta Accreta in Patients With Scarred Uterus: A Retrospective Cohort Study. Front Med (Lausanne). 2019;6:289.

23. Boroomand Fard M, Kasraeian M, Vafaei H, Jahromi MA, Arasteh P, Shahraki HR, et al. Introducing an efficient model for the prediction of placenta accreta spectrum using the MCP regression approach based on sonography indexes: how efficient is sonography in diagnosing accreta? BMC Pregnancy Childbirth. 2020 Feb 17;20(1):111.

24. Moons KGM, Altman DG, Reitsma JB, Ioannidis JPA, Macaskill P, Steyerberg EW, et al. Transparent Reporting of a multivariable prediction model for Individual Prognosis or Diagnosis (TRIPOD): explanation and elaboration. Ann Intern Med. 2015 Jan 6;162(1):W1-73.

25. Yang D, Dalton JE. A unified approach to measuring the effect size between two groups using SAS(r). $2012 ; 6$.

26. Austin PC. Using the standardized difference to compare the prevalence of a binary variable between two groups in observational research. Communications in Statistics: Simulation and Computation. 2009;38(6):1228-34.

27. Debray TPA, Vergouwe Y, Koffijberg H, Nieboer D, Steyerberg EW, Moons KGM. A new framework to enhance the interpretation of external validation studies of clinical prediction models. J Clin Epidemiol. 2015 Mar;68(3):279-89.

28. Altman DG, Bland JM. Diagnostic tests 3: receiver operating characteristic plots. BMJ. 1994 Jul $16 ; 309(6948): 188$.

29. Hosmer D, Lemeshow S. Applied Logistic Regression, 2nd Ed.Chapter 5. New York, NY: John Wiley and Sons, 2000. Pp. 160-164.

30. Van Calster B, Nieboer D, Vergouwe Y, De Cock B, Pencina MJ, Steyerberg EW. A calibration hierarchy for risk models was defined: from utopia to empirical data. J Clin Epidemiol. 2016;74:167-76. 
31. Steyerberg EW, Vergouwe Y. Towards better clinical prediction models: seven steps for development and an ABCD for validation. Eur Heart J. 2014 Aug 1;35(29):1925-31.

32. Vickers AJ, Van Calster B, Steyerberg EW. Net benefit approaches to the evaluation of prediction models, molecular markers, and diagnostic tests. BMJ. 2016 Jan 25;352:i6.

33. Maher MA, Abdelaziz A, Bazeed MF. Diagnostic accuracy of ultrasound and MRI in the prenatal diagnosis of placenta accreta. Acta Obstet Gynecol Scand. 2013 Sep;92(9):1017-22.

34. Meng X, Xie L, Song W. Comparing the diagnostic value of ultrasound and magnetic resonance imaging for placenta accreta: a systematic review and meta-analysis. Ultrasound Med Biol. 2013 Nov;39(11):1958-65.

35. Daney de Marcillac F, Moliere S, Pinton A, Weingertner A-S, Fritz G, Viville B, et al. [Accuracy of placenta accreta prenatal diagnosis by ultrasound and MRI in a high-risk population]. J Gynecol Obstet Biol Reprod (Paris). 2016 Feb;45(2):198-206.

36. Einerson BD, Rodriguez CE, Silver RM, Donnelly MA, Kennedy AM, Woodward PJ. Accuracy and Interobserver Reliability of Magnetic Resonance Imaging for Placenta Accreta Spectrum Disorders. Am J Perinatol. 2020 Jan 27;

37. Silver RM, Landon MB, Rouse DJ, Leveno KJ, Spong CY, Thom EA, et al. Maternal morbidity associated with multiple repeat cesarean deliveries. Obstet Gynecol. 2006 Jun;107(6):1226-32.

38. Kayem G, Seco A, Beucher G, Dupont C, Branger B, Crenn Hebert C, et al. Clinical Profiles of Placenta Accreta Spectrum: the PACCRETA Population-Based Study. BJOG. 2021 Jan 3;

39. Vergouwe Y, Steyerberg EW, Eijkemans MJC, Habbema JDF. Substantial effective sample sizes were required for external validation studies of predictive logistic regression models. J Clin Epidemiol. 2005 May;58(5):475-83.

Figure legends:

Figure 1: Flowchart showing the constitution patients at risk for placenta accreta spectrum in (A) the Weiniger development cohort, (B) validation cohort A and (C) validation cohort B; HHMC: Hadassah Hebrew University Medical Center; BWH: Brigham and Women's Hospital CUIMC: Columbia University Irving Medical Center; CD: Caesarean Delivery, PAS: Placenta Accreta Spectrum; US: Ultrasound.

Figure 2: Receiver Operating Characteristic curves of showing discrimination of the model in (A) the Weiniger development cohort, $(\mathrm{B})$ validation cohort $\mathrm{A}$ and $(\mathrm{C})$ validation cohort $\mathrm{B}$.

Figure 3: Calibration of the model in the (A) Weiniger development cohort, (B) validation cohort A and (C) validation cohort $\mathrm{B}$.

Figure 4: Decision curve analyses showing plots of net benefit vs threshold probability for the (A) Weiniger development cohort, $(\mathrm{B})$ validation cohort $\mathrm{A}$ and $(\mathrm{C})$ validation cohort $\mathrm{B}$. Net benefit is the net true positive classification rate (calculation described in Appendix S2). Threshold probability is the minimum predicted probability of the outcome at which the clinician would change the management of a patient (Appendix S2).

\section{Hosted file}

Table 1.pdf available at https://authorea.com/users/406396/articles/517073-a-predictionmodel-for-placenta-accreta-spectrum-a-multicentre-external-validation-study

\section{Hosted file}

Table 2.pdf available at https://authorea.com/users/406396/articles/517073-a-predictionmodel-for-placenta-accreta-spectrum-a-multicentre-external-validation-study

\section{Hosted file}


Table 3.pdf available at https://authorea.com/users/406396/articles/517073-a-predictionmodel-for-placenta-accreta-spectrum-a-multicentre-external-validation-study

\section{Hosted file}

Table 4.pdf available at https://authorea.com/users/406396/articles/517073-a-predictionmodel-for-placenta-accreta-spectrum-a-multicentre-external-validation-study

\section{Hosted file}

Figure 1.pdf available at https://authorea.com/users/406396/articles/517073-a-predictionmodel-for-placenta-accreta-spectrum-a-multicentre-external-validation-study

\section{Hosted file}

Figure 2.pdf available at https://authorea.com/users/406396/articles/517073-a-predictionmodel-for-placenta-accreta-spectrum-a-multicentre-external-validation-study

\section{Hosted file}

Figure 3.pdf available at https://authorea.com/users/406396/articles/517073-a-predictionmodel-for-placenta-accreta-spectrum-a-multicentre-external-validation-study

\section{Hosted file}

Figure 4.pdf available at https://authorea.com/users/406396/articles/517073-a-predictionmodel-for-placenta-accreta-spectrum-a-multicentre-external-validation-study 